(c) American Dairy Science Association, 2004.

\title{
Development of an Optimal Index to Improve Lactation Yield and Persistency with the Least Selection Intensity
}

\author{
K. Togashi ${ }^{1}$ and C. Y. Lin ${ }^{2, *}$ \\ ${ }^{1}$ National Agricultural Research Center for Hokkaido Region, \\ 1 Hitsujigaoka, Toyohiraku, Sapporo, Japan 062-8555 \\ ${ }^{2}$ Dairy and Swine Research and Development Centre, \\ Agriculture and Agri-Food, Canada
}

age of cow, gestation, dry period, rations, season, temperature, and humidity (Schmidt and Van Vleck, 1974). It is of interest to separate environmental factors from genetic factors controlling the lactation curve to develop effective strategies to manipulate the genetic aspect of the lactation curve. Although there are different measures of persistency according to the review of Swalve and Gengler (1999), persistency commonly refers to the rate of decline after peak yield. A cow with a flatter curve is said to be more persistent. A more persistent cow is more efficient in roughage usage, under less stress during peak yield, and is more resistant to stressrelated disease (Zimmermann and Sommer, 1973; Sölkner and Fuchs, 1987). Dekkers et al. (1998) reported that the economic benefit of persistency depends highly on lactation length and calving interval. They estimated the economic value of persistency to be about $5 \%$ of the economic value of milk production with an average calving interval of 12.4 mo as compared with an economic value of persistency of around $15 \%$ of production with an average calving interval of $13 \mathrm{mo}$.

Genetic correlations between first lactation milk yield and persistency depend on the measures of persistency used (Swalve and Gengler, 1999; Jakobsen et al., 2002). Two equivalent selection procedures for simultaneous improvement of lactation milk yield and persistency (Lin and Togashi, 2002; Togashi and Lin, 2003) have been presented: index selection based on stage EBV and index selection based on random regression (RR) coefficients from a test day model (Schaeffer and Dekkers, 1994; Jamrozik et al., 1997). Both procedures were developed to achieve prespecified stage gains. However, there are many possible lactation curves that could satisfy the prespecified stage gains, especially when each stage is long. Therefore, it is important to develop an "ideal" index to fulfill the prespecified stage gains with the lowest selection intensity. The objective of this study was to develop a selection index to realize the prespecified stage gains with the lowest selection intensity. A numerical example is given to demonstrate the approach.

Received January 27, 2004

Accepted April 20, 2004.

Corresponding author: K. Togashi; e-mail: tkenji@naro.affrc.go.jp.

* Present address: Department of Animal and Poultry Science, University of Guelph, Ontario, Canada N1G 2W1. 


\section{MATERIALS AND METHODS}

A RR test day model based on Legendre polynomials was used in the development of this study.

\section{Selection Intensity}

Correlated response for day i across lactation ( $i=5$, $6, \ldots, 305)$ because of conventional selection on lactation EBV was estimated as $\underline{\Delta}_{\mathrm{c}}=\mathbf{G}_{\mathbf{L}}\left(\overline{\mathrm{i}} / \sigma_{\mathrm{EBV}_{\mathrm{L}}}\right)$, where $\underline{\Delta}_{\mathrm{c}}$ is a vector of correlated responses from d 5 to $305, \mathbf{G}_{\mathrm{L}}$ is a $(301 \times 301)$ genetic covariance matrix of daily yields, $\mathbf{1}$ is a summing vector of 1 with dimension equal to the number of days from DIM $5 \sim 305, \bar{i}$ is the intensity of selection, and $\sigma_{\mathrm{EBV}_{\mathrm{L}}}$ is the standard deviation of lactation EBV. $\sigma_{\mathrm{EBV}}$ is estimated as $\sqrt{\mathbf{1}^{\prime} \Phi \mathbf{K} \Phi^{\prime} \mathbf{1}}$, where $\mathbf{K}$ is

the $k \times k$ genetic covariance matrix of $\mathrm{RR}$ coefficients with $(k-1)$ being the order of polynomials fitted, $\Phi$ is a $301 \times k$ matrix of Legendre polynomials evaluated from DIM $5 \sim 305$. The new lactation curve after one cycle of selection on 305-d milk is the sum of the original lactation curve before selection and correlated responses for each day of the lactation.

Index selection based on stage EBV (I), (Lin and Togashi, 2002; Togashi and Lin, 2003) with $(k-1)$ orders of Legendre polynomials and $s$ stages is

$$
\mathrm{I}=\sum_{\mathrm{j}=1}^{\mathrm{s}} \mathrm{b}_{\mathrm{j}} \mathrm{EBV}_{\mathrm{j}}=\sum_{\mathrm{j}=1}^{\mathrm{s}} \mathrm{b}_{\mathrm{j}} \sum_{\mathrm{t}=\mathrm{m}_{\mathrm{j}}}^{\mathrm{n}_{\mathrm{j}}} \sum_{\mathrm{i}=0}^{\mathrm{k}-1} \phi_{\mathrm{i}}(t) \alpha_{\mathrm{i}}
$$

where $b_{\mathrm{j}}$ is the index weight for stage $\mathrm{j}, \phi_{\mathrm{i}}(t)$ is order $\mathrm{i}$ of the Legendre polynomial evaluated at day $t$ standardized, $\alpha_{\mathrm{i}}$ is order $\mathrm{i}$ of the RR coefficient, and $\mathrm{m}_{\mathrm{j}}$ and $\mathrm{n}_{\mathrm{j}}$ are the first and last day of stage $\mathrm{j}$, respectively. In matrix notation, $\mathrm{I}=\mathbf{b}^{\prime} \boldsymbol{\theta}$, where $\boldsymbol{\theta}$ is a column vector containing the EBV of $s$ stages and $\mathbf{b}$ is a vector of index weights. The expected genetic gains for the $s$ stages because of selection on I are $\boldsymbol{\Delta}=\mathbf{G} \mathbf{b}\left(\overline{\mathrm{i}} / \sigma_{\mathrm{I}}\right)$, where vector $\Delta$ is the expected genetic gains for $s$ stages $\left(\Delta G_{1}, \Delta G_{2}\right.$, $\left.\ldots, \Delta G_{\mathrm{s}}\right), \mathbf{G}$ is the genetic covariance matrix of $s$ stages, and $\sigma_{\mathrm{I}}$ is the standard deviation of I. Selection intensity (i) required to achieve $\underline{\Delta}$ can be obtained by setting $\overline{\mathrm{i}}=$ $\sigma_{\mathrm{I}}$. Therefore,

$$
\begin{gathered}
\mathbf{b}=\mathbf{G}^{-1} \underline{\Delta} \\
\text { and } \sigma_{\mathrm{I}}^{2}=\operatorname{var}\left(\mathbf{b}^{\prime} \boldsymbol{\theta}\right)=\mathbf{b}^{\prime} \mathbf{G} \mathbf{b}=\underline{\Delta^{\prime}} \mathbf{G}^{-1} \underline{\Delta}
\end{gathered}
$$

Selection index based on RR coefficients $\left(I^{*}\right)$ is described as

$$
I^{*}=b_{0}^{*} \alpha_{0}+b_{1}^{*} \alpha_{1}+b_{2}^{*} \alpha_{2}+\ldots+b_{\mathrm{k}-1}^{*} \alpha_{\mathrm{k}-1}=\alpha^{\prime} \mathbf{b}^{*} .
$$

Index weights $\left(\mathbf{b}^{*}\right)$ are given by $\mathbf{b}^{*}=\mathbf{K}^{-1} \underline{\Delta}_{\alpha}$, where $\Delta_{\alpha}$ is a $(\mathrm{k} \times 1)$ vector containing the difference in $\mathrm{RR}$ coefficients before and after selection. Thus, selection intensity required to achieve $\boldsymbol{\Delta}_{\alpha}$ is obtained as $\overline{\mathrm{i}}=\sigma_{I^{*}}$ where

$$
\sigma_{\mathrm{I}^{*}}^{2}=\operatorname{var}\left(\boldsymbol{\alpha}^{\prime} \mathbf{b}^{*}\right)=\mathbf{b}^{* \prime} \mathbf{K} \mathbf{b}^{*}=\underline{\Delta}_{\alpha}^{\prime} \mathbf{K}^{-1} \underline{\Delta}_{\alpha}
$$

\section{Minimizing Selection Intensity of an Index to Achieve the Intended Stage Gains}

Given a fixed order of $R R$ coefficients and a fixed set of stage genetic gains $(\boldsymbol{\Delta})$, different combinations of selection differentials on each order of $R R$ coefficients $\left(\underline{\Delta}_{\alpha_{\mathrm{i}}}\right)$ could satisfy these 2 conditions. An interesting question is which combination is optimal so that it would require the lowest selection intensity of an index to achieve the prespecified stage gains. Lin (1985) showed that 3 different restricted indexes were derived to satisfy the same restriction by placing different emphasis on the index traits, suggesting that given a set of prespecified restriction, the solution to achieve the restriction is not unique. Therefore, there are different sets of $\underline{\Delta}_{\alpha_{\mathrm{i}}}(i=1,2 \ldots)$ that would satisfy a prespecified vector of stage gains $\Delta^{*}$. Different indexes derived from different sets of $\underline{\Delta}_{\alpha_{\mathrm{i}}}(i=1,2 \ldots)$ which satisfy the same vector of intended stage gains would have different selection intensities. Our goal is to find a vector $\underline{\Delta}_{\alpha_{\mathrm{k}}}$ to construct an index that yields the minimum selection intensity and satisfies the prespecified constraint.

Togashi and Lin (2003) showed that when $\bar{i}=\sigma_{I^{*}}$, the vector of expected genetic gains of stages is $\underline{\Delta}=$ $\mathbf{S} \underline{\Delta}_{\alpha_{\mathbf{k}}}$ where $\mathbf{S}$ is a $(s \times k)$ matrix that contains the sum of each order of Legendre polynomials within stages, i.e.,

$$
\mathbf{S}=\left[\begin{array}{cccc}
\mathrm{n}_{1} & \mathrm{n}_{1} & & \mathrm{n}_{1} \\
\sum_{\mathrm{t}=\mathrm{m}_{1}} \phi_{0}(t) & \sum_{\mathrm{t}=\mathrm{m}_{1}} \phi_{1}(t) & \ldots & \sum_{\mathrm{t}=\mathrm{m}_{1}} \phi_{\mathrm{k}-1}(t) \\
\sum_{\mathrm{n}=\mathrm{m}_{2}} \phi_{0}(t) & \sum_{\mathrm{t}=\mathrm{m}_{2}} \phi_{1}(t) & \ldots & \sum_{\mathrm{t}=\mathrm{m}_{2}} \phi_{\mathrm{k}-1}(t) \\
\vdots & \vdots & \ddots & \vdots \\
\mathrm{n}_{\mathrm{s}} & \mathrm{n}_{\mathrm{s}} & & \vdots \\
\sum_{\mathrm{t}=\mathrm{m}_{\mathrm{s}}} \phi_{0}(t) & \sum_{\mathrm{t}=\mathrm{m}_{\mathrm{s}}} \phi_{1}(t) & \ldots & \sum_{\mathrm{t}=\mathrm{m}_{\mathrm{s}}} \phi_{\mathrm{k}-1}(t)
\end{array}\right] .
$$

Lagrange multiplier was used to choose a vector $\underline{\Delta}_{\alpha_{\mathrm{k}}}$ so that the index constructed based on $\underline{\Delta}^{*}$ has a 
minimum variance with the restriction that the vector of expected stage genetic gains is equal to the vector of intended genetic gains $\left(\Delta^{*}=\left[\Delta G_{1}^{*} \Delta G_{2}^{*} \cdots \Delta G_{s}^{*}\right]^{\prime}\right)$.

The function to be minimized is $f=\underline{\Delta}_{\alpha_{\mathrm{k}}} \mathbf{K}^{-1} \underline{\Delta}_{\alpha_{\mathrm{k}}}+$ $\underline{\boldsymbol{\lambda}}^{\prime}\left[\mathbf{S} \underline{\Delta}_{\alpha_{\mathrm{k}}}-\underline{\Delta}^{*}\right]$, where $\underline{\boldsymbol{\lambda}}=\left[\begin{array}{llll}\lambda_{1} & \lambda_{2} & \ldots & \lambda_{\mathrm{s}}\end{array}\right]^{\prime}$ is a vector of Lagrange multipliers.

Setting the partial derivatives of $f$ with respect to $\underline{\Delta}_{\alpha_{\mathrm{k}}}$ equal to zero leads to

$$
\frac{\partial \mathrm{f}}{\partial \underline{\Delta}_{\alpha_{\mathrm{k}}}}=2 \mathbf{K}^{-1} \underline{\Delta}_{\alpha_{\mathrm{k}}}+\mathbf{S}^{\prime} \underline{\boldsymbol{\lambda}}=\underline{\mathbf{0}}
$$

Setting the partial derivatives of $f$ with respect to $\underline{\lambda}$ equal to zero leads to

$$
\frac{\partial \mathrm{f}}{\partial \underline{\lambda}}=\mathbf{S} \underline{\Delta}_{\alpha_{\mathrm{k}}}-\underline{\Delta^{*}}=\underline{\mathbf{0}} .
$$

Equations [4] and [5] can be written jointly as follows:

$$
\left[\begin{array}{ccc}
2 \mathbf{K}^{-1} & \vdots & \mathbf{S}^{\prime} \\
\ldots & \vdots & \ldots \\
\mathbf{S} & \vdots & \mathbf{0}
\end{array}\right]\left[\begin{array}{c}
\underline{\Delta}_{\alpha_{\mathrm{k}}} \\
\ldots \\
\underline{\boldsymbol{\lambda}}
\end{array}\right]=\left[\begin{array}{c}
\underline{\mathbf{0}} \\
\ldots \ldots \\
\underline{\boldsymbol{\Delta}^{*}}
\end{array}\right] .
$$

According to the principle of Lagrange multiplier, the solution vector $\underline{\Delta}_{\alpha_{\mathrm{k}}}$ in equation [6] would lead to minimum selection intensity and satisfy the constraints of the expected genetic gains being equal to the intended genetic gains.

The inverse of the coefficient matrix of equation [6] can be obtained through inversion by partitioning (Searle, 1966). Therefore, the solution to equation [6] is

$$
\left[\begin{array}{c}
\underline{\Delta}_{\alpha_{\mathrm{k}}} \\
\ldots \\
\underline{\boldsymbol{\lambda}}
\end{array}\right]=\left[\begin{array}{ccc}
\frac{1}{2}\left[\mathbf{K}-\mathbf{K S}^{\prime}(\mathbf{S K S})^{\prime}\right)^{-1} \mathbf{S K} & \vdots & \mathbf{K S} \mathbf{S}^{\prime}\left(\mathbf{S K S}^{\prime}\right)^{-1} \\
\ldots \ldots \ldots \ldots \ldots . . & \vdots & \ldots \ldots \ldots . . \\
\left(\mathbf{S K S}^{\prime}\right)^{-1} \mathbf{S K}^{\prime} & \vdots & -2\left(\mathbf{S K S}^{\prime}\right)^{-1}
\end{array}\right]\left[\begin{array}{c}
\underline{\mathbf{0}} \\
\ldots \ldots \\
\underline{\Delta}^{*}
\end{array}\right]
$$

The first set of equations is equal to

$$
\underline{\Delta}_{\alpha_{\mathrm{k}}}=\mathbf{K S}^{\prime}\left(\mathbf{S K S}^{\prime}\right)^{-1} \underline{\Delta}^{*} .
$$

Index coefficients $\left(\mathbf{b}^{*}\right)$ for the selection index based on RR coefficients are determined by $\mathbf{b}^{*}=\mathbf{K}^{-1} \underline{\Delta}_{\alpha}$. Finally, the index based on $R R$ coefficients that would achieve the prespecified stage gains with the lowest selection intensity is

$$
\mathrm{I}^{*}=\alpha^{\prime} \mathbf{b}^{*}=\alpha^{\prime} \mathbf{K}^{-1} \underline{\Delta}_{\alpha_{\mathrm{k}}}=\alpha^{\prime} \mathbf{S}^{\prime}\left(\mathbf{S K S}^{\prime}\right)^{-1} \underline{\Delta} *
$$

This is a general case whether the number of stages $(s)$ is equal to that of RR coefficients fitted $(k)$. When $s=k$, equation [7] reduces to $\underline{\Delta}_{\alpha_{\mathrm{k}}}=\mathbf{S}^{-1} \underline{\Delta}$ and, subsequently, I* $=\alpha^{\prime} \mathbf{K}^{-1} \mathbf{S}^{-1} \Delta^{*}$. The correlated response for DIM $\mathrm{i}\left(\Delta \mathrm{C}_{\mathrm{i}}\right)$ from selection on $\mathrm{I}^{*}$ is given by $\Delta C_{i}=$ $\underline{\Delta}_{\alpha_{\mathbf{k}}}^{\prime}\left(\phi_{0}\left(i^{*}\right) \phi_{1}\left(i^{*}\right) \ldots \phi_{k-1}\left(i^{*}\right)\right)^{\prime}$, where $i^{*}$ is standardized time.

\section{Relationship Between I and I* with the Lowest Selection Intensity}

Togashi and Lin (2003) showed that a selection index based on RR coefficients ( $I^{*}=\alpha^{\prime} \mathbf{b}^{*}$ ) is equivalent to a selection index based on stage $\mathrm{EBV}\left(\mathrm{I}=\boldsymbol{\theta}^{\prime} \mathbf{b}\right)$ and that vector $\mathbf{b}$ is equivalent to $\left(\mathbf{S S}^{\prime}\right)^{-1} \mathbf{S b}^{*}$. These 2 equalities hold true whether these 2 indexes result in minimum selection intensity. When $\mathbf{b}^{*}$ used to construct index I* leads to a minimum selection intensity as shown in the preceding section, then $\mathbf{b}=\left(\mathbf{S S}^{\prime}\right)^{-1} \mathbf{S b}^{*}$ used to construct index I will also lead to a minimum selection intensity because $\left(\mathbf{S S}^{\prime}\right)^{-1} \mathbf{S}$ is a constant independent of the calculation of $\mathbf{b}$ and $\mathbf{b}^{*}$. Therefore, $\mathbf{b}$ and $\mathbf{b}^{*}$ can be easily converted from each other.

Note that $\mathbf{b}=\left(\mathbf{S S}^{\prime}\right)^{-1} \mathbf{S b}^{*}=\left(\mathbf{S S}^{\prime}\right)^{-1} \mathbf{S S}^{\prime}\left(\mathbf{S K S}^{\prime}\right)^{-1} \boldsymbol{\Delta}^{*}=$ $\left(\mathbf{S K S}^{\prime}\right)^{-1} \underline{\Delta}^{*}=\mathbf{G}^{-1} \underline{\Delta}^{*}$. This shows that index I, which was converted from index I* with minimum selection intensity, also has the least intensity of selection.

\section{Numerical Example}

As an example, a lactation length of $305 \mathrm{~d}$ was partitioned into 3 stages $(s=3$ ): stage 1 (DIM $5 \sim 65$ ), stage 2 (DIM $66 \sim 280$ ), and stage 3 (DIM $281 \sim 305$ ). Assume that the annual genetic gain on a lactation basis is 100 $\mathrm{kg} \mathrm{EBV}$ and that we want to reduce stage 1 by $25 \mathrm{~kg}$ EBV and to improve stage 2 by $125 \mathrm{~kg}$ EBV while holding stage 3 to zero change. This means that $\Delta^{*}=$ $\left[\begin{array}{lll}-25 & 125 & 0\end{array}\right]^{\prime}$.

The genetic covariance matrix $(\mathbf{K})$ of $R R$ coefficients $(k=5)$ estimated by Pool et al. (2000) based on Dutch test-day data was used to compute the proposed index:

$\mathbf{K}_{5 \times 5}=$
$\left[\begin{array}{rrrrr}9.98598 & 0.19091 & -1.21056 & 0.47058 & -0.21283 \\ 0.19091 & 0.94982 & -0.11455 & -0.07786 & 0.04629 \\ -1.21056 & -0.11455 & 0.33542 & -0.09034 & 0.02855 \\ 0.47058 & -0.07786 & -0.09034 & 0.08828 & -0.04342 \\ -0.21283 & 0.04629 & 0.02855 & -0.04342 & 0.03586\end{array}\right]$

As defined previously, matrix $\mathbf{S}_{3 \times 5}$ contains the sum of each order of Legendre polynomials within each of the 3 stages. 


$$
\begin{gathered}
\mathbf{S}_{3 \times 5}= \\
{\left[\begin{array}{rrrrr}
43.134 & -59.767 & 46.360 & -18.564 & -6.691 \\
152.028 & 31.598 & -75.332 & -8.688 & -11.208 \\
17.678 & 28.169 & 30.558 & 27.252 & 20.043
\end{array}\right] .}
\end{gathered}
$$

According to equation [7],

$$
\underline{\Delta}_{\alpha_{5}}=\mathbf{K S}^{\prime}\left(\mathbf{S K S}^{\prime}\right)^{-1} \underline{\Delta^{*}}
$$

$$
=\left[\begin{array}{llllll}
0.47443 & 0.27291 & -0.59536 & 0.08922 & -0.01560
\end{array}\right]^{\prime} \text {. }
$$

Index coefficients $\left(\mathbf{b}^{*}\right)$ of $\mathrm{I}^{*}$ with the lowest selection intensity are

$$
\begin{aligned}
& \mathbf{b}^{*}=\mathbf{K}^{-1} \underline{\Delta}_{\alpha_{5}}= \\
& {\left[\begin{array}{lllll}
-0.29107 & -0.06789 & -3.06742 & -1.12771 & -0.99830
\end{array}\right]^{\prime}}
\end{aligned}
$$

Finally, I* $=\boldsymbol{\alpha}^{\prime} \mathbf{b}^{*}=-0.29107 \alpha_{0}-0.06789 \alpha_{1}-3.06742 \alpha_{2}$ $-1.12771 \alpha_{3}-0.99830 \alpha_{4}$. Selection intensity $(\overline{\mathrm{i}})$ to realize the prespecified stage gains $\left(\Delta^{*}=\left[\begin{array}{lll}-25 & 125 & 0\end{array}\right]^{\prime}\right)$ is

$$
\bar{i}=\sigma_{I^{*}}=\sqrt{\underline{\Delta}^{\prime}{ }_{\alpha_{5}} \mathbf{K}^{-1} \underline{\Delta}_{\alpha_{5}}^{\prime}}=1.259 \text {. }
$$

The correlated response for DIM i $\left(\Delta \mathrm{C}_{\mathrm{i}}\right)$ is $\Delta C_{i}=\underline{\Delta}^{\prime} \alpha_{5}$

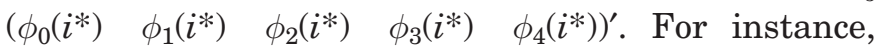
the correlated response for DIM $65\left(\Delta \mathrm{C}_{65}\right)$ is

$$
\begin{aligned}
& \Delta \boldsymbol{C}_{65}=\underline{\Delta}_{\alpha_{5}}^{\prime}\left(\phi_{0}(-0.6) \quad \phi_{1}(-0.6) \quad \phi_{2}(-0.6)\right. \\
& \left.\phi_{3}(-0.6) \quad \phi_{4}(-0.6)\right)^{\prime} \\
& =\left(\begin{array}{lllll}
0.47443 & 0.27291 & -0.59536 & 0.08922 & -0.01560
\end{array}\right) \\
& \times\left(\begin{array}{lllll}
0.70711 & -0.73485 & 0.06325 & 0.67350 & -0.86550
\end{array}\right)^{\prime} \\
& =0.17086 \text {. }
\end{aligned}
$$

The conversion of $b *$ to $b$ for the index based on stage EBV with the least selection intensity gives $\mathbf{b}=$ $\left(\mathbf{S S}^{\prime}\right)^{-1} \mathbf{S b}^{*}=\left[\begin{array}{lll}-0.01797 & 0.00908 & -0.05073\end{array}\right]^{\prime}$.

Selection intensity $(\overline{\mathrm{i}})$ for index $\mathrm{I}$ is $\overline{\mathrm{i}}=\sigma_{\mathrm{I}}=\sqrt{\mathbf{b}^{\prime} \mathbf{G} \mathbf{b}}=$ 1.259. Clearly, selection intensity of 1.259 was the same for both I and I* because both indexes are equivalent.

Next, consider selection index ( $\left.\mathrm{I}^{* *}\right)$ based on RR coefficients with no restriction imposed on the selection intensity (Togashi and Lin, 2003).

$$
\underline{\Delta}_{\alpha_{5}}=\left(\mathbf{S}^{\prime} \mathbf{S}\right)^{-1} \mathbf{S}^{\prime} \underline{\Delta}^{*}=
$$

$\left[\begin{array}{llllll}0.47581 & 0.71104 & -0.29458 & -0.43750 & -0.37500\end{array}\right]^{\prime}$

Index coefficients for $I^{* *}$ are

$$
\begin{aligned}
& \mathbf{b}^{* *}=\mathbf{K}^{-1} \underline{\Delta}_{\alpha_{5}}= \\
& {\left[\begin{array}{lllll}
-0.15053 & -0.67550 & -7.18392 & -35.70532 & -47.99197
\end{array}\right]^{\prime} \text {, }}
\end{aligned}
$$

and selection intensity $(\overline{\mathrm{i}})$ is $\overline{\mathrm{i}}=\sigma_{\mathrm{I}^{* *}}=\sqrt{\left(\mathbf{b}^{* *}\right)^{\prime} \mathbf{K}^{* * *}}=$ 5.931

Correlated response for DIM i $\left(\Delta \mathrm{C}_{\mathrm{i}}\right)$ is equal to $\Delta C_{i}=$

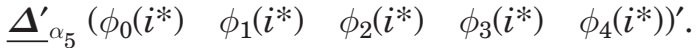

For instance,

$$
\begin{aligned}
& \Delta \mathrm{C}_{65}=\underline{\Delta}_{\alpha_{5}}^{\prime}\left(\phi_{0}(-0.6) \quad \phi_{1}(-0.6) \phi_{2}(-0.6)\right. \\
& \left.\phi_{3}(-0.6) \quad \phi_{4}(-0.6)\right)^{\prime} \\
& =\left(\begin{array}{lllll}
0.47581 & 0.71104 & -0.29458 & -0.43750 & -0.37500
\end{array}\right) \\
& \times\left(\begin{array}{lllll}
0.70711 & -0.73485 & 0.06325 & 0.67350 & -0.86550
\end{array}\right)^{\prime} \\
& =-0.17478 \text {. }
\end{aligned}
$$

$\sigma_{\mathrm{EBV}}$ for conventional selection based on 305-d milk $\mathrm{EBV}$ is

$$
\sigma_{\mathrm{EBV}_{\mathrm{L}}}=\sqrt{\mathbf{1}^{\prime} \boldsymbol{\prime} \mathbf{K} \boldsymbol{\Phi}^{\prime} \mathbf{1}}=671.95 .
$$

Selection intensity $(\overline{\mathrm{i}})$ to achieve $100 \mathrm{~kg}$ total milk yield is $\overline{\mathrm{i}}=100 / \sigma_{\mathrm{EBV}_{\mathrm{L}}}=0.149$. Correlated response $\left(\underline{\Delta}_{\mathrm{c}}\right)$ for each DIM because of conventional selection is

$$
\underline{\Delta}_{\mathrm{c}}=\mathbf{G} \mathbf{1}\left(\overline{\mathrm{i}} / \sigma_{\mathrm{EBV}_{\mathrm{L}}}\right) \text {. }
$$

\section{RESULTS AND DISCUSSION}

Lin (1985) showed that different forms of restricted indexes could be derived to satisfy the same restriction. The more severe the restriction that is imposed, the higher the selection intensity that is needed to realize the restriction. Of all possible indexes that satisfy the same prespecified constraint, it is critical to choose the one with the lowest selection intensity for practical application because the lower the selection intensity, the greater the probability of realizing the selection goal. This study showed that although 3 indexes, $I^{* *}, I^{*}$, and I, satisfy the same preconditions, the selection intensity for I** is much larger than that for I* or I (5.931 vs. 1.259). This confirms that different sets of indexes may satisfy the same preconditions imposed on genetic gains between stages, but there is only one unique index based on equation [7] that satisfies the preconditions and minimizes the selection intensity.

Togashi and Lin (2003) showed $\boldsymbol{\Delta}_{\alpha}=\left(\mathbf{S}^{\prime} \mathbf{S}\right)^{-1} \mathbf{S}^{\prime} \boldsymbol{\Delta}$. The index derived based on $\underline{\Delta}_{\alpha}$ would realize the prespecified stage gains, but there is no guarantee that the index would offer the lowest selection intensity possible. 


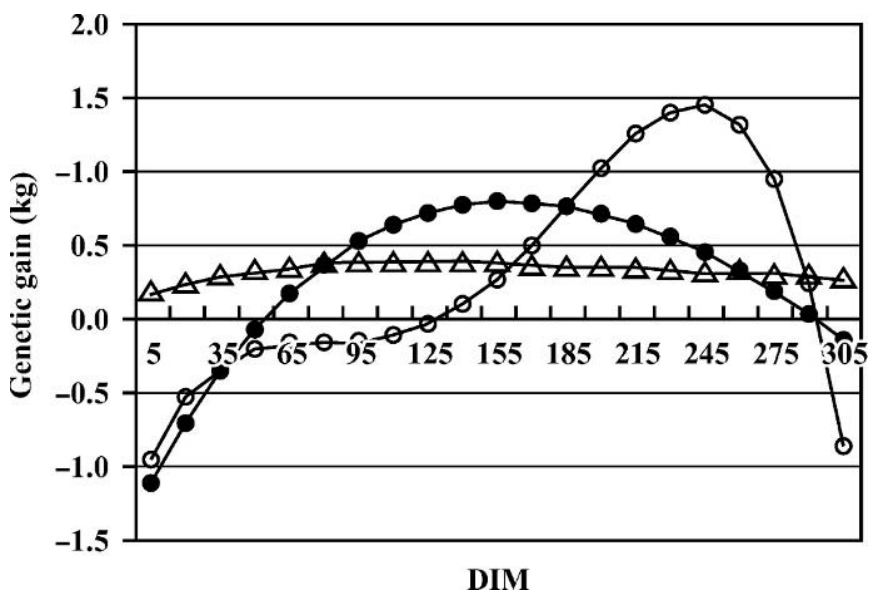

Figure 1. Genetic gains from I(or I*), I**, and 305-d milk yield ( $\mathrm{I}=$ stage of gain index, $\mathrm{I}^{*}=$ index based on random regression $[\mathrm{RR}]$ coefficients with the least selection intensity, $\mathrm{I}^{* *}=$ index based on $R R$ coefficients with no restriction on selection intensity). Legend: $\boldsymbol{Q}=\mathrm{I}$ or I*; $\bigcirc=\mathrm{I}^{* *}$; and $\Delta=$ selection based on lactation $\mathrm{EBV}_{\mathrm{L}}$.

Their approach would result in an index with minimum selection intensity only if the number of stages partitioned is equal to the number of RR coefficients fitted $(s=k)$. In contrast, the index resulting from $\underline{\Delta}_{\alpha_{\mathrm{k}}}$ in equation [7] of this study provides a unique solution to achieve the prespecified stage gains with minimum selection intensity.

The correlated daily responses across lactation for those I (or I*), I**, and selection based on 305-d lactation milk $\left(\mathrm{EBV}_{\mathrm{L}}\right)$ in numerical example are shown in Figure 1. The prespecified stage gains for the 3 indexes, $\mathrm{I}, \mathrm{I}^{*}$, and $\mathrm{I}^{* *}$, are $-25,125$, and $0 \mathrm{~kg}$ EBV for stage 1 (DIM $5 \sim 65$ ), stage 2 (DIM $66 \sim 280$ ), and stage 3 (DIM $281 \sim 305$ ), respectively. The daily genetic response because of selection on I** (without restriction on selection intensity) changes more drastically than that caused by selection on $\mathrm{I}\left(\mathrm{I}^{*}\right)$ or $\mathrm{EBV}_{\mathrm{L}}$. The fluctuating pattern of daily genetic responses for I** would yield a larger genetic deviation across the lactation and require a higher selection intensity than I ( $\left.I^{*}\right)$ to achieve the selection goal. Daily genetic gains because of selection on $\mathrm{EBV}_{\mathrm{L}}$ are almost at the same level throughout the lactation because selection on $\mathrm{EBV}_{\mathrm{L}}$ imposes no restriction and have no control over daily genetic responses.

Given the same genetic improvement of $100 \mathrm{~kg}$ EBV in this study, conventional selection on $\mathrm{EBV}_{\mathrm{L}}$ requires a selection intensity of 0.149 as compared with 1.259 for I (or $\mathrm{I}^{*}$ ) or 5.931 for $\mathrm{I}^{* *}$. The index with restriction requires higher selection intensity because the imposition of desired gains between different lactation stages reduces genetic advance (genetic cost of restriction), thus requiring higher selection intensity to achieve the same amount of improvement as the conventional selec- tion for lactation EBV. The additional selection intensity needed for desired gain index varies depending upon the lactation response assumed a priori $(100 \mathrm{~kg}$ EBV in the example), the number of lactation stages partitioned, the length of each lactation stage, and most importantly, how the desired genetic gains are distributed between different stages of lactation. Given a fixed lactation response to be allocated between different stages of lactation, selection intensity increases with increasing severity of the restriction. Therefore, great caution must be taken in imposing desired genetic gains. Otherwise, selection intensity required may be too high to be sustained by the population.

In the numerical example, prespecified absolute gains for the 3 lactation stages are assumed to be $\Delta^{*}=$ $\left[\begin{array}{lll}-5 & 125 & 0\end{array}\right]^{\prime}$, which is equal to $\Delta^{*}=5\left[\begin{array}{ll}-1 & 25^{-}\end{array}\right]^{\prime}$. Note that the common factor of 5 can be dropped without affecting the proportionality of the index (b or $\mathbf{b}^{*}$ ). Therefore, the imposition of prespecified absolute gains is in fact equivalent to the restriction of prespecified relative changes of $\Delta^{*}=\left[\begin{array}{lll}-1 & 25 & 0\end{array}\right]^{\prime}$. However, prespecified absolute gains of $\Delta^{*}=\left[\begin{array}{lll}-5 & 125 & 0\end{array}\right]^{\prime}$ with a total of $100 \mathrm{~kg}$ EBV should be used to ensure a fair comparison with conventional selection for lactation response of $100 \mathrm{~kg}$ EBV in the numerical example.

\section{CONCLUSIONS}

This study showed that different restricted indexes (e.g., I, I*, and I**) exist to satisfy the same precondition of improving lactation yield and persistency. Lagrange multiplier approach was used to develop an index (I*) to realize the prespecified stage gains with the lowest selection intensity. To achieve the same prespecified restriction, optimal index I* or I requires a selection intensity of 1.259 as compared with 5.931 for nonoptimal index I**. In contrast, conventional selection based on 305-d lactation EBV with no restriction requires a selection intensity of 0.149 to achieve the same genetic gain of $100 \mathrm{~kg}$ EBV as do I* or I**. Therefore, selection intensity increases with increasing severity of the restriction. Given the same level of restriction, the lower the selection intensity required for a selection criterion, the greater the probability of realizing the selection goal. Daily genetic gains across lactation fluctuate drastically for nonoptimal index I** as compared with optimal index I or I*. Two alternative equations were developed to convert between indexes I* and I. Conventional selection based on 305-d EBV does not impose restriction on the genetic changes between stages and shows rather constant daily genetic gains across lactation. 


\section{ACKNOWLEDGMENTS}

The authors thank M. H. Pool for kindly providing the genetic covariance matrix of the random regression coefficients for this study.

\section{REFERENCES}

Dekkers, J. C. M., J. H. Ten Haag, and A. Weersink. 1998. Economic aspects of persistency of lactation in dairy cattle. Livest. Prod. Sci. 53:237-252.

Jakobsen, J. H., P. Madsen, J. Jensen, J. Pedersen, L. G. Christensen, and D. A. Sorensen. 2002. Genetic parameters for milk production and persistency for Danish Holsteins estimated in random regression models using REML. J. Dairy Sci. 85:1607-1616.

Jamrozik, J., L. R. Schaeffer, and J. C. M. Dekkers. 1997. Genetic evaluation of dairy cattle using test day yields and random regression model. J. Dairy Sci. 80:1217-1226.

Lin, C. Y. 1985. A simple stepwise procedure of deriving selection index with restrictions. Theor. Appl. Genet. 70:147-150.
Lin, C. Y., and K. Togashi. 2002. Simultaneous improvement of lactation milk and persistency. 7th WCGALP CD-ROM Commun. \# 09-05.

Pool, M. H., L. L. G. Janss, and T. H. E. Meuwissen. 2000. Genetic parameters of Legendre polynomials for first parity lactation curves. J. Dairy Sci. 83:2640-2649.

Schaeffer, L. R., and J. C. M. Dekkers. 1994. Random regressions in animal models for test-day production in dairy cattle. Proc. 5th WCGALP 18:443-446.

Schmidt, G. H., and L. D. Van Vleck. 1974. Principles of Dairy Science. Freeman and Co., San Francisco, CA.

Searle, S. R. 1966. Matrix Algebra for the Biological Science. John Wiley \& Sons, Inc., New York.

Sölkner, J., and W. Fuchs. 1987. A comparison of different measures of persistency with special respect to variation of test-day milk yields. Livest. Prod. Sci. 16:305-319.

Swalve, H. H., and N. Gengler. 1999. Genetics of lactation persistency. Occ. Publ. Br. Soc. Anim. Sci. 24:75-82.

Togashi, K., and C. Y. Lin. 2003. Modifying the lactation curve to improve lactation milk and persistency. J. Dairy Sci. 86:14871493.

Zimmermann, E., and H. Sommer. 1973. Zum Laktationsverlauf von Kühen in Hochleistungsherden und dessen Beeinflussung durch nichterbliche Faktoren. Züchtungskunde. 45:75-88. 